Ann. Zootech., I973, $22(2), 243-247$.

NOTE

\title{
COMPOSITION EN ACIDES AMINÉS DU LAIT DE TRUIE
}

\author{
P.-H. DUÉE et J. JUNG \\ avec la collaboration technique de A. M. Guevgneau \\ Station de Recherches sur l'Élevage des Porcs \\ Centre national de Recherches zootechniques, I. N. R. A., \\ 78350 Jouy en Josas
}

\section{RÉSUMÉ}

La composition en acides aminés des protéines du colostrum et du lait de I 2 truies Large-White a été déterminée par dosage chromatographique.

Les teneurs moyennes, exprimées en $\mathrm{g}$ p. I $6 \mathrm{~g}$ d'azote, respectivement pour le colostrum et le lait, sont les suivantes :

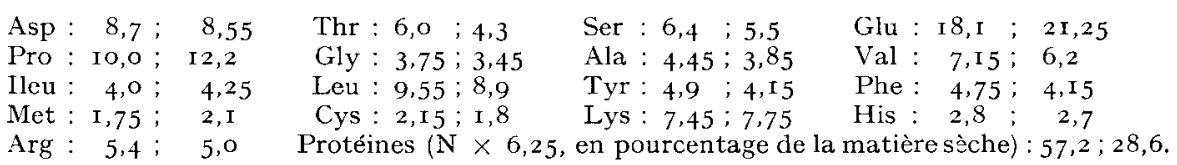

Par rapport au lait, le colostrum est plus riche en 9 acides aminés, la thréonine en particulier. Par contre, la méthionine, l'acide glutamique et la proline ont des concentrations plus élevées dans le lait.

Les résultats individuels font apparaître de faibles coefficients de variation pour chacune des concentrations en acides aminés considérées.

\section{INTRODUC'TION}

Dans les conditions pratiques d'élevage, le lait maternel représente, pour le jeune porcelet, la seule source alimentaire, tout au moins pendant les deux premières semaines de lactation. L'étude de la composition en acides aminés du lait de truie permettra d'estimer l'apport de ces nutriments en relation avec le gain de poids des porcelets pendant cette période. Les variations de la composition du lait de truie, en fonction du stade de lactation sont bien connues. (Salmon-Legagneur, I964). En particulier, le taux azoté, après une brusque augmentation suivant la mise bas, décroît progressivement pour se maintenir à un niveau constant vers le 
Ioe jour de lactation, avant d'augmenter légèrement après six semaines. De ce fait, il apparaît deux stades préférentiels dans l'étude de la composition des protéines du lait : d'une part, au début de la lactation (qui correspond à la production de colostrum dont on sait qu'il contient une quantité importante d'immunoglobulines), d'autre part, vers le Io $^{\mathrm{e}}$ jour de lactation.

Peu d'auteurs ont, jusqu'à présent, porté intérêt à la détermination de la composition en acides aminés du lait de truie (Beacom et Bowland, 1951; Cuperlović, 1967; Elliott et al., 1971). Les résultats rapportés sont incomplets et procèdent soit d'une technique autre que celle actuellement utilisée (dosage microbiologique), soit d'un très faible nombre d'animaux.

\section{MATÉRIEL E'T MÉTHODES}

L'étude a été entreprise sur I 2 truies Large-White, dont le numéro de portée était, en moyenne, de 4. Le poids des animaux après la mise bas était de $203,5 \pm 5,7 \mathrm{~kg}$, le gain net de poids pendant la gestation précédente s'élevait à $29,2 \mathrm{~kg}$, auquel succéda une perte de poids pendant la lactation (35 jours) de I I, $7 \mathrm{~kg}$.

Les performances zootechniques des animaux pendant la lactation sont rassemblées dans le tableau suivant (tabl. I).

TABLEAU I

Performances zootechniques des animaux

\begin{tabular}{|c|c|}
\hline Nombre de porcelets nés par portée & $11,3 \pm 0,6(1)$ \\
\hline Poids moyen à la naissance (kg) .. & $1,15 \pm 0,04$ \\
\hline Nombre de porcelets à 21 jours. & $8,4 \pm 0,4$ \\
\hline Poids moyen à 21 jours $(\mathrm{kg}) \ldots$ & $5,0^{\prime} \pm 0,21$ \\
\hline Nombre de porcelets au sevrage $(35 \mathrm{j})$ & $8,3 \pm 0,4$ \\
\hline Poids moyen des porcelets au sevrage (kg) & $8,24 \pm 0,41$ \\
\hline
\end{tabular}

(1) Eicart-type de la moyenne.

Les animaux reçoivent le même régime standard en gestation (régime à base d'orge, de tourteau de soja et de farine de luzerne) et en lactation (aliment à base d'orge, de tourteau de soja, de farine de poisson et de farine de luzerne). Le niveau alimentaire est en gestation et en lactation respectivement, de 2,5 et $5,9 \mathrm{~kg}$ par jour, ce qui satisfait les besoins nutritionnels des truies, définis par le N. R. C. (I968), pour les stades physiologiques concernés.

- La récolte des échantillons de lait est donc effectuée à 2 stades bien définis : d'une part, dans les I 2 heures après la mise bas (colostrum), d'autre part, Io jours après.

L'éjection de lait est provoquée par injection intraveineuse d'ocytocine (dose employée : Io UI). Les quantités recueillies manuellement varient de 100 à $200 \mathrm{ml}$. La composition chimique moyenne du colostrum et du lait ainsi collectés figure dans le tableau 2.

- Les échantillons de colostrum et de lait ont subi deux hydrolyses acides 24 et 48 heures (en présence de $\mathrm{HCl}(6 \mathrm{~N})$ ), puis les acides aminés des hydrolysats sont dosés par la méthode de Moore et al. (I958), utilisant la technique de chromatographie sur colonne échangeuse d'ions.

Le tryptophane, totalement détruit lors de l'hydrolyse acide, n'est pas dosé ; les acides aminés soufrés (méthionine et cystine) sont oxydés au préalable par l'acide performique et dosés par la même méthode que précédemment.

En comparant les résultats de la composition en acides aminés du lait, obtenus après hydrolyse soit de $24 \mathrm{~h}$ soit de $48 \mathrm{~h}$, il apparaît que les différentes concentrations dans les deux hydrolysats sont semblables, sauf pour la thréonine, la sérine et la tyrosine dont les teneurs sont significativement plus élevées dans l'hydrolysat de 24 heures, et pour l'isoleucine et la valine, difficiles à libérer et dont les teneurs sont significativement plus élevées dans l'hydrolysat de 
48 heures. En conséquence, dans les résultats définitifs, il n'a été tenu compte que des valeurs obtenues avec l'hydrolysat de 24 heures, pour la thréonine, la sérine et la tyrosine, avec l'hydrolysat de 48 heures, pour l'isoleucine et la valine; pour tous les autres acides aminés, ces résultats sont les moyennes des valeurs obtenues après deux temps d'hydrolyse.

Il a été procédé, pour l'ensemble des données, à une comparaison des moyennes par la méthode des couples, sur les ro truies pour lesquelles nous avons obtenu des données complètes concernant le colostrum et le lait.

\section{TABLEAU 2}

Composition chimique du colostrum et du lait de truie

(en $\mathrm{g}$ p. $100 \mathrm{~g}$ de lait frais)

\begin{tabular}{|c|c|c|}
\hline Stade de lactation & Colostrum & 10 jours \\
\hline $\begin{array}{c}\text { Matière sèche } \\
\text { Matières azotées totales } \\
(\mathrm{N} \times 6,25) \\
\text { Matières minérales }\end{array}$ & $\begin{array}{c}22,65 \pm 0,67\left(^{1}\right) \\
12,75 \pm 0,75 \\
0,69 \pm 0,02\end{array}$ & $\begin{array}{r}19,18 \pm 0,57 \\
5,33 \pm 0,10 \\
0,73 \pm 0,04\end{array}$ \\
\hline
\end{tabular}

(1) Moyenne \pm écart-type.

\section{RÉSULTATS ET DISCUSSION}

La composition en acides aminés du colostrum et du lait de truie est présentée dans le tableau 3 .

A un stade donné, les résultats des concentrations en acides aminés du lait de truie, exprimés en grammes d'acide aminé pour $\mathbf{r} 6 \mathrm{~g}$ d'azote, font apparaître une variabilité peu élevée de ces teneurs : le coefficient de variation des différentes concentrations oscille entre 2 et 8 p. Ioo.

Si les protéines du colostrum et du lait de truie sont bien pourvues en acides aminés indispensables, et en particulier en lysine, elles semblent pourtant, relativement pauvres en acides aminés soufrés (méthionine, surtout) ce qui avait déjà été montré par ailleurs (BEAcom et BowLAND, I95I ; Cuperlović, I967; Ellioti et al., I97I).

L'utilisation d'un nombre assez important d'animaux permet de mettre en évidence des différences significatives entre les deux stades de lactation. Par rapport au lait, le colostrum est plus riche en 9 acides aminés, la thréonine en particulier, mais également la valine, la leucine, la phénylalanine, la tyrosine, la cystine, l'alanine, la sérine, et l'arginine. Par contre, le lait de truie comporte par rapport au colostrum, des teneurs plus élevées en méthionine, acide glutamique et proline. Les concentrations des autres acides aminés ne subissent pas de variations entre les deux stades de lactation.

Ces différences reflètent les modifications intervenant dans les proportions des diverses fractions azotées du lait. D'après les résultats de SALmon-LEgagneur (1964), la fraction azotée constituée des globulines (support des propriétés immunitaires du colostrum) est remplacée, partiellement et assez rapidement, par le groupe des caséines. Or, les immunoglobulines contiennent des taux de thréonine, valine, leucine et à moindre degré de cystine, alanine et sérine, plus élevés que les caséines. Au contraire, celles-ci sont plus riches en acide glutamique, proline et méthionine (Tristram et Sмiтh, 1963). 
TABLEAU 3

Teneurs en acides aminés du colostrum et du lait de truie

(en g p. I6 g N)

\begin{tabular}{|c|c|c|c|}
\hline & Colostrum & Lait de 10 jours & $\begin{array}{l}\text { Signification } \\
\text { statistique de } \\
\text { la différence }\left({ }^{\mathbf{1}}\right)\end{array}$ \\
\hline Acide aspartique ... & $8,7 \pm 0,1\left(^{2}\right)$ & $8,55 \pm 0,10\left(^{2}\right)$ & NS \\
\hline Thréonine ....... & $6,0 \pm 0,2$ & $4,3 \pm 0,1$ & $* * *$ \\
\hline Sérine.......... & $6,4 \pm 0,1$ & $5,5 \pm 0,1$ & $* * *$ \\
\hline Acide glutamique. . & $18,1 \pm 0,3$ & $21,25 \pm 0,20$ & $* * *$ \\
\hline Proline & $10,0 \pm 0,2$ & $12,2 \pm 0,2$ & $* * *$ \\
\hline Glycine . . . . . . & $3,75 \pm 0,05$ & $3,45 \pm 0,05$ & $* *$ \\
\hline Alanine ........ & $4,45 \pm 0,07$ & $3,85 \pm 0,04$ & $* * *$ \\
\hline Valine $\ldots . . . \ldots$ & $7,15 \pm 0,16$ & $6,2 \pm 0,1$ & $* * *$ \\
\hline Isoleucine $\ldots \ldots \ldots$ & ${ }_{4,00} \pm 0,05$ & $4,25 \pm 0,05$ & $* *$ \\
\hline Leucine . . . . . . . & $9,55 \pm 0,10$ & $8,9 \pm 0,1$ & $* * *$ \\
\hline Tyrosine ....... & $4,9 \pm 0,1$ & $4,15 \pm 0,06$ & $* * *$ \\
\hline Phénylalanine.... & $4,75 \pm 0,07$ & $4,15 \pm 0,04$ & $* * *$ \\
\hline Méthionine ...... & $1,75 \pm 0,0_{4}^{\prime}$ & $2,10 \pm 0,05$ & $* * *$ \\
\hline Cystine $\ldots \ldots \ldots$ & $2,15 \pm 0,07$ & $1,80 \pm 0,03$ & $* *$ \\
\hline Lysine $\ldots . . . \ldots$ & $7,45 \pm 0,08$ & $7,75 \pm 0,12$ & * \\
\hline Histidine $\ldots \ldots \ldots$ & $2,80 \pm 0,03$ & $2,70 \pm 0,05$ & NS \\
\hline Arginine ..... & $5,4 \pm 0,1$ & $5,0 \pm 0,1$ & $* *$ \\
\hline $\begin{array}{r}\text { Somme des AA } \\
\text { indispensables et } \\
\text { semi-indisp...... }\end{array}$ & 55,9 & 51,3 & \\
\hline M. A. $(\%$ MS) & 57,2 & 28,6 & \\
\hline
\end{tabular}

(1) Signification statistique de la différence; NS :non significatif ; * : différence significative au seuil $\mathrm{P}<0,05 ; * *$ : différence significative au seuil $\mathrm{P}<0,01 ; * * *$ : différence significative au seuil $\mathrm{P}<0,001$.

(2) Ecart-type de la moyenne (12 échantillons pour le colostrum; 10 échantillons pour le lait).

Enfin, la comparaison des compositions en acides aminés des protéines du lait de truie et du lait de vache, d'après Pion et Fauconneau (I966), fait ressortir de plus f.ibles teneurs en acides aminés indispensables et semi-indispensables dans le lait de truie, en particulier pour l'isoleucine, la valine, les acides aminés aromatiques (tyrosine et phénylalanine) et, secondairement, la lysine et la méthionine. Par contre, l'arginine et 1 cystine ont des concentrations plus élevées dans les protéines du lait de truie.

En ce qui concerne les acides aminés non indispensables, de plus fortes teneurs en proline et en glycine sont à remarquer dans le lait de truie.

L'analyse de la composition en acides aminés du lait de truie permet de confirmer la valeur biologique élevée de ses protéines. Il apparâ̂t d'autre part que cette qualité des protéines est peu 
affectée par des variations individuelles, tout au moins dans l'échantillon de truies observées. Finalement, plus que la qualité, la quantité des protéines dans le lait, donc la production laitière, reste le facteur limitant de la croissance des porcelets sous la mère.

Reçu pour publication en janvier 1973.

\section{SUMMARY}

\section{AMINO ACID COMPOSITION OF SOW'S MILK}

The amino acid composition of colostrum and milk proteins from 12 Large-White sows was determined by means of chromatographic analysis.

The mean contents, expressed in $\mathrm{g}$ per $16 \mathrm{~g}$ nitrogen were the following for colostrum and milk, respectively :

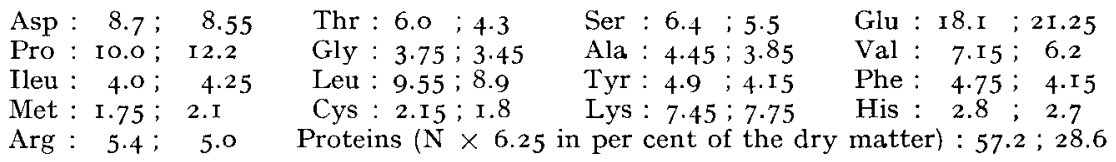

Compared with milk, colostrum contained higher amounts of 9 amino acids, in particular threonine. On the other hand, contents of methionine, glutamic acid and proline were higher in milk.

According to the individual results, the variation coefficients for each amino acid concentration considered were small.

\section{RÉFÉRENCES BIBLIOGRAPHIQUES}

Beacom S. E., Bowland J. P., I95 I. The essential amino acid (except tryptophan) content of colostrum and milk of the sow. $J . N u t r ., 45,419-429$.

Cupkrlovic M., I 967 . Free Amino Acids in the blood plasma of newborn piglets before and after the first intake of colostrum. Acta vet. scand., 8, 217-227.

Elliott R. F., Vander Noot G. W., Gilbreath R. L., Fisher H., I97I. Effect of dietary protein level on composition changes in sow colostrum and milk. J. Anim. Sci. 32, II28-Ir37.

MoORE S., Spackman D. H., Stein W. H., I958. Chromatography of Amino Acids on sulfonated polystyrene Resins. Anal. Chem., 30, I 8 5-I 190.

N. R. C., I958. National Research Council. Nutrient Requivements of domestic animals 2. Nutrient require:nents of swine. National Academy of Sciences Pub. $\mathrm{n}^{0}$ I599. Washington, D. C., U. S. A.

Pion R., Fauconneau G., 1966. Les acides aminés des protéines alimentaires. Méthodes de dosage et résultats obtenus. Cahier A. E. C., n ${ }^{\circ} 6$, I57-I75.

Sadmon-Legagneur E., I964. Les constituants azotés du lait de truie : évolution au cours de la lactation et izfluence du régime alimentaire. Ann. Biol. anim. Bioch. Biophys., 4, 49-6z.

TristiziM G. R., Sмith R. H., 1963. The amino acid composition of some purified proteins. In Advanzes in protein chemistry, 18, 227-318, Acad. Press. New York, London. 